\title{
Replacing Metal Parts with Resin in Pipe Installations and Gas Equipment
}

\author{
Yuji Higuchi ${ }^{*}$ and Kazuhisa Igawa \\ Osaka Gas Co., Ltd., Osaka 541-0046, Japan
}

\begin{abstract}
This report examines the transition from metal to resin occurring in gas distribution facilities and gas equipment parts. Japan's low-pressure steel gas pipelines have transitioned to resin, with polyethylene currently accounting for $40 \%$ of all low-pressure underground pipes. A comprehensive polyethylene piping system is taking shape as the resin trend spreads to couplers and valves. The switch from metal to resin solves corrosion problems in gas pipes. Furthermore, polyethylene piping systems are more resistant to earthquakes, providing a more stable gas supply. Resin is also beginning to replace metal in gas equipment parts. The switch first occurred in pipe parts that carry both hot and cold water. Now, brass fittings, headers and steel pipes are being replaced with PPS (Polyphenylene sulfide). Resin used for hot water is exposed to harsh conditions. Determining whether resin is a suitable replacement for metal requires knowledge of the various resin materials' characteristics, standardized assessments to determine whether those parts are being used in a suitable environment, and appropriate testing methods. We conducted water-hammer tests, constant load creep tests and other forms of experiments under water in high-temperature and high-pressure environments on metal parts and their resin counterparts to assess resin's potential as a replacement.
\end{abstract}

Key words: Resin, gas distribution facility, gas equipment, metal replacement, lightweight, cost reduction.

\section{Introduction}

The movement to replace metal parts with resin in automobiles and other industrial products is gaining traction [1-6]. Replacing metal parts with resin results in lighter weight and a reduction in costs. It has other benefits: corrosion and earthquake resistance. In automobiles, better fuel efficiency can be expected. In other industrial products, resin has contributed to lower manufacturing and shipping costs. This trend toward resin has spread to consumer electronics and gas equipment parts.

In this report, we will look at how resin is beginning to replace metal for gas distribution facilities and gas equipment parts and its suitability in piping. In recent years, parts that have been already switched out to resin are now being considered for replacement with even cheaper resin. We will address that in this report as well.

*Corresponding author: Yuji Higuchi, research fields: polymer physical properties evaluation. E-mail: higuchi@osakagas.co.jp.

\subsection{Switching Gas Distribution Facilities to Resin}

Fig. 1 shows the west European countries that made changes to their pipe material [7]. Around 2000, a majority of pipes were still made of metal. In recent years, that has dropped to about half. Plastic is expected to make up a majority of pipe material in the future.

In Japan, low-pressure gas pipelines are being switched from steel to polyethylene. Fig. 2 shows the different types of gas piping. Low-pressure pipes are moving from steel and cast iron, through resin-lined steel, and then to polyethylene.

Fig. 3 shows the switch to polyethylene pipes in Japan [8]. Polyethylene pipes are increasing annually. They currently account for $40 \%$ of low-pressure underground pipes.

In addition to pipes, resin is spreading to couplers and valves, contributing to a comprehensive polyethylene piping system. One such example is the switch in valve material, shown in Fig. 4. 


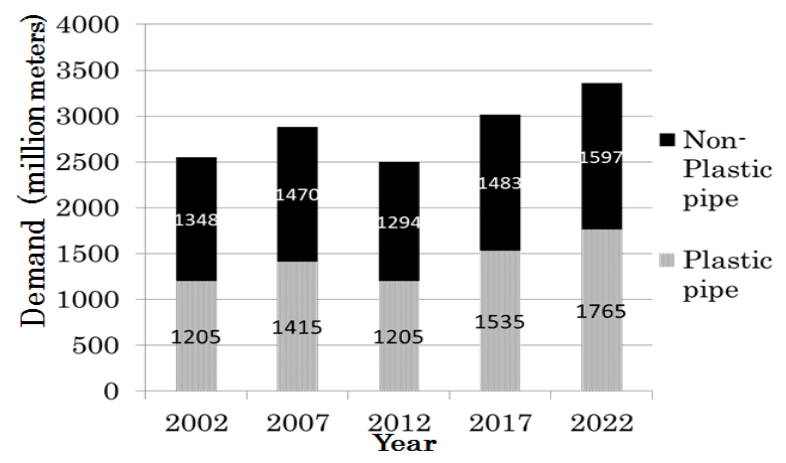

Fig. 1 Changes in pipe material in western Europe.

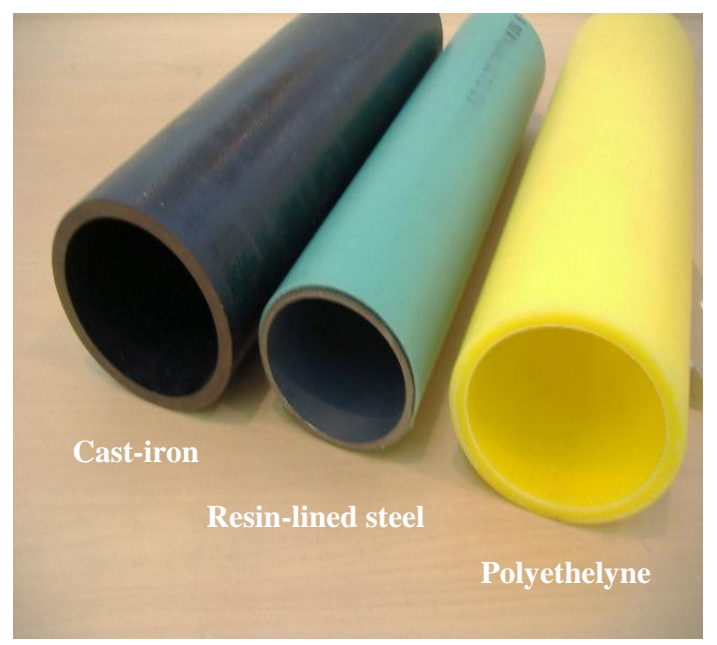

Fig. 2 Changes in gas piping in Japan.

The switch to polyethylene from metal takes care of corrosion and earthquake concerns. Consequently, one can say resin contributes to a stable and reliable supply of low-pressure piping. During the 2011 great East-Japan earthquake, the polyethylene gas pipes remained undamaged. Japan's goal is to spread polyethylene so that it makes up $60 \%$ of all pipes by 2030 .

Fig. 5 shows the different types of plastic pipes in western Europe. PVC (Polyvinyl chloride), which was the fastest-growing plastic piping material, still dominates the region, but the options are growing [1]. In earthquake-prone Japan, polyethylene has been the material of choice over PVC pipe because of its superior flexibility. But Japan can consider options other than polyethylene in the future.

\subsection{The Use of Resin in Gas Equipment Parts}

Along with gas distribution facilities, gas equipment parts are also being phased out of metal and in to resin. The switch from metal to resin helps reduce the cost of the gas equipment itself and makes the construction work flow easier. The transition to plastic - in particular, a specific type of plastic with reliable performance in the auto industry is taking place with gas equipment parts. With gas equipment parts, the switch to resin happened in piping that carries hot and cold water. Cross-linked polyethylene and polybutene

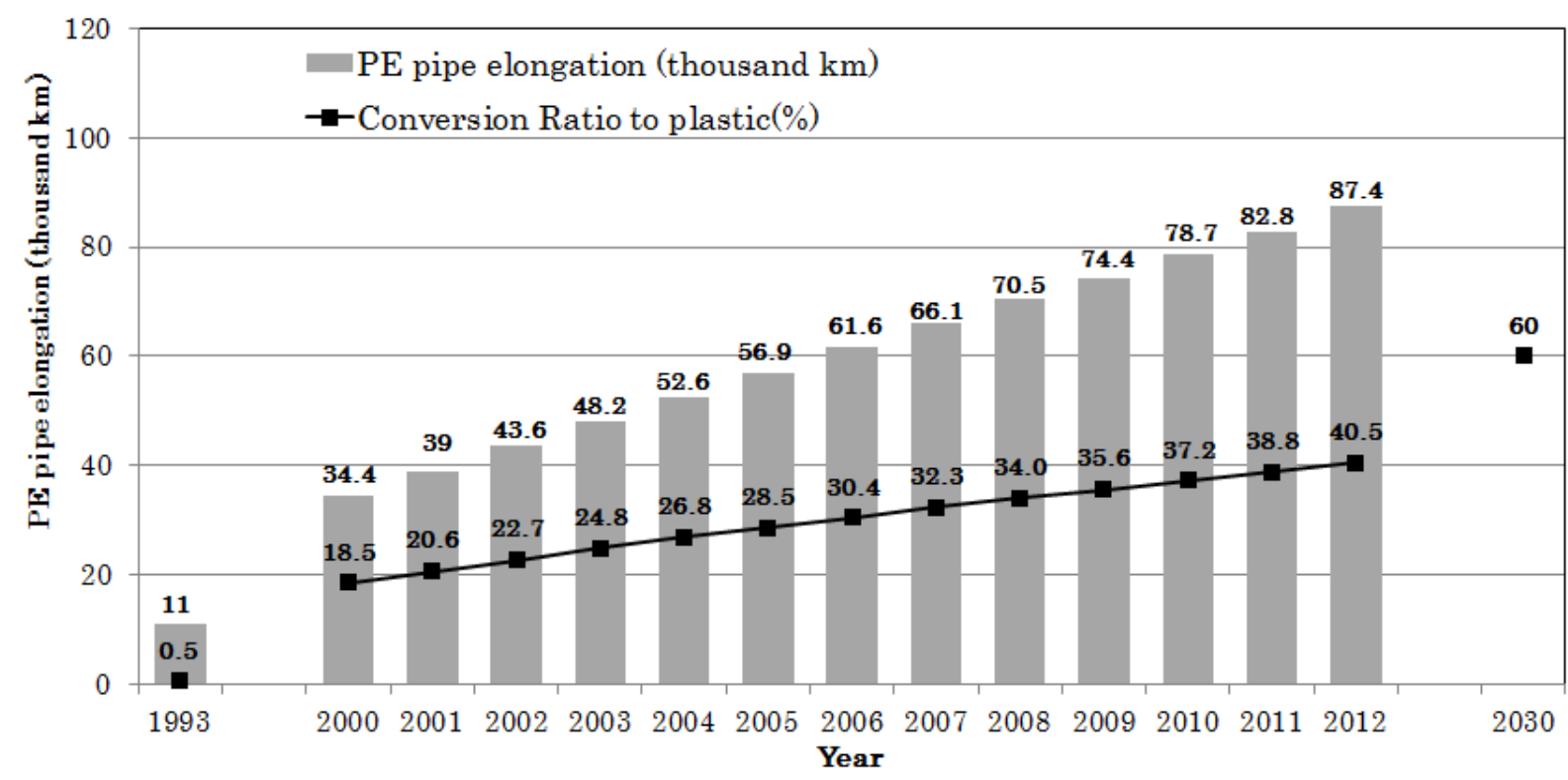

Fig. 3 The spread of polyethylene pipes in Japan. 


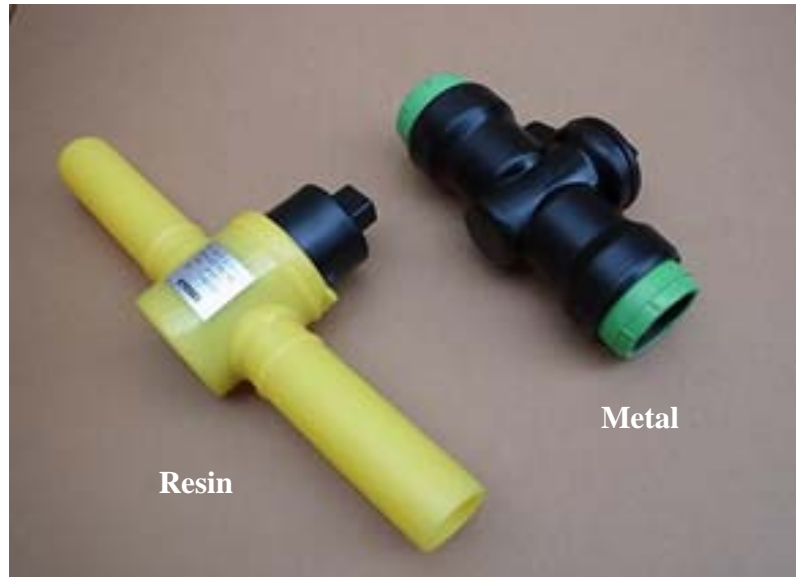

Fig. 4 Switch in gas valves.

pipes are examples of the plastic pipes. Fig. 6 shows the switch from metal to plastic pipes. Fig. 7 shows the couplers; Fig. 8, the headers. Brass couplers and headers are also being replaced with PPS (Polyphenylene sulfide), a leading engineering plastic material.

Fig. 9 shows the different classifications of resin. When used as a metal substitute, resin must be especially heat resistant. General-purpose plastic is low cost and is used in everyday goods but is heat-resistant only up to $100{ }^{\circ} \mathrm{C}$. A higher grade of resin is general engineering plastic, which is heat-resistant up to $150^{\circ} \mathrm{C}$. For more heat resistance, there's the super engineering plastic, which withstands heat of above $150{ }^{\circ} \mathrm{C}$. That makes super engineering plastic the best candidate as a metal substitute.

Restrictions prevent Japan from using resin for flammable parts or parts through which gas emissions pass. Outside of Japan, however, resin is being used for gas equipment. Fig. 10 demonstrates how resin parts are used in hot-water heaters. Fan casings, combustion air guides, emission ducts, emission tops and other such parts are already made from resin. Switching the internal parts of a hot-water heater to resin will create a more reliable product because of its light weight and lower cost. In these areas, Japan has room to consider deregulation.

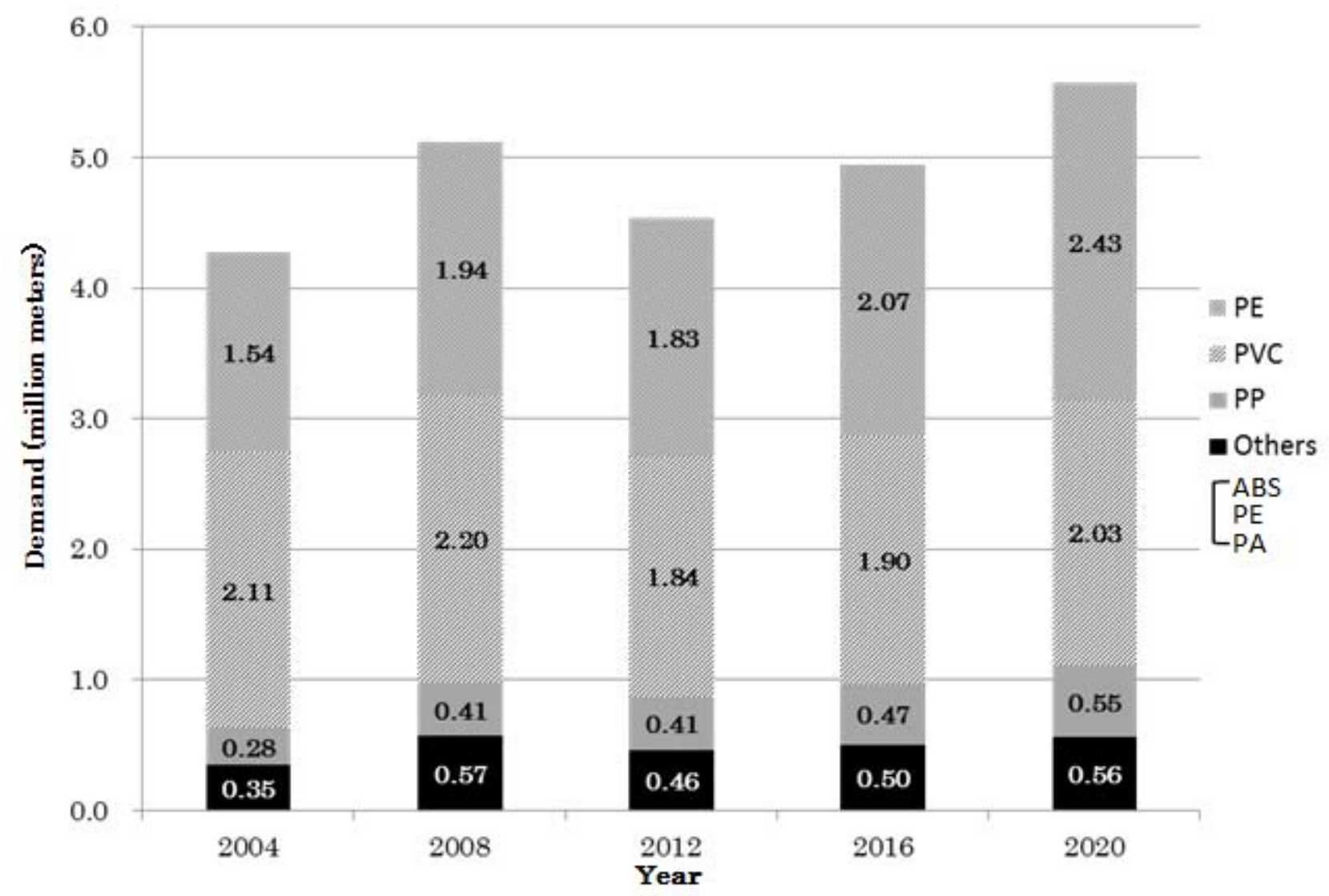

Fig. 5 Different types of plastic pipes in western Europe. 


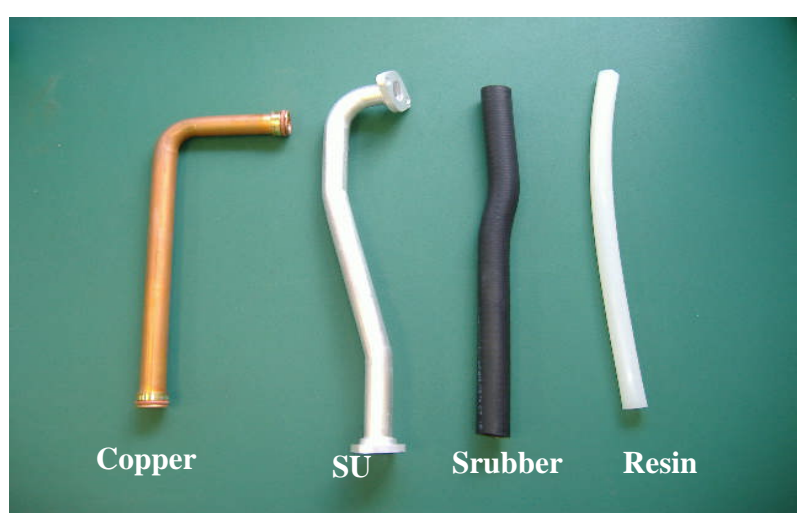

Fig. 6 Changes in hot-water piping.

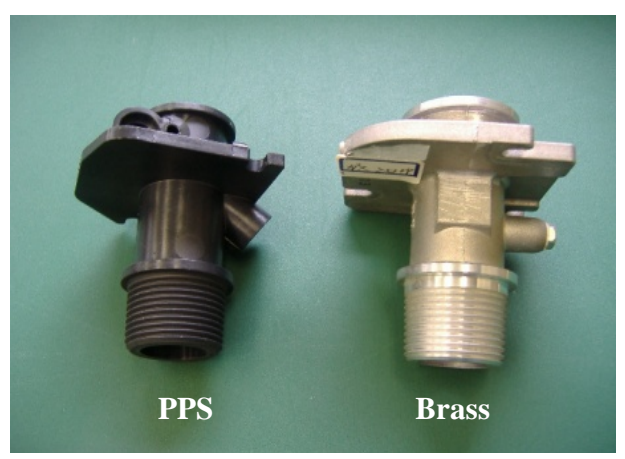

Fig. 7 Changes in pipe fittings.

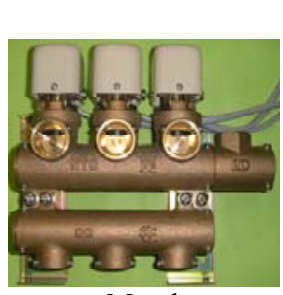

Metal

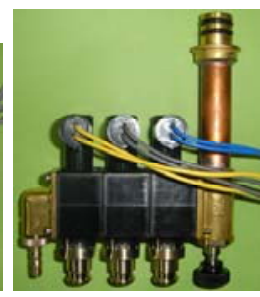

Mixture

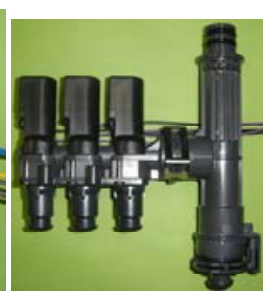

PPS
Fig. 8 The switch in headers.

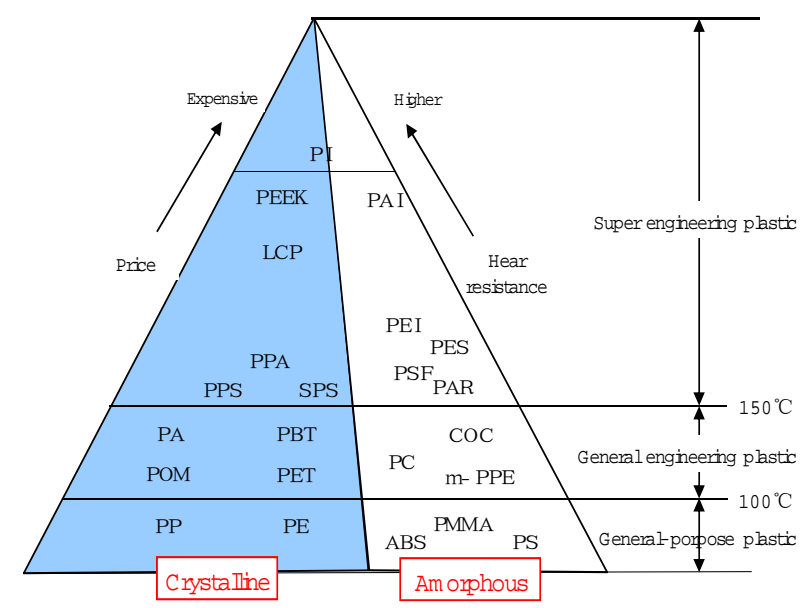

Fig. 9 Classifications of resin.

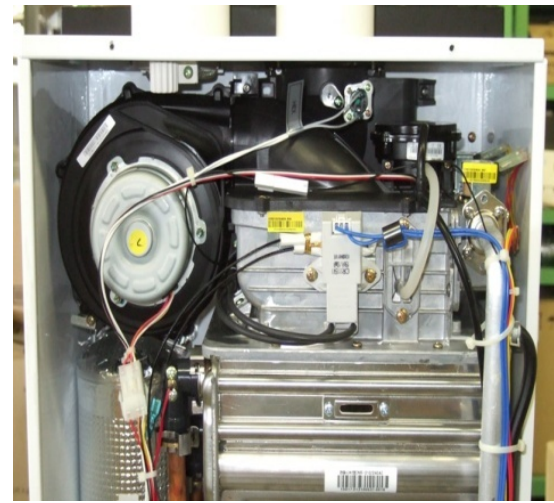

Fig. 10 Example of resin used in foreign hot-water dispensers.

\section{Materials and Methods}

\subsection{Assessing the Characteristics of Resin}

The standards used to assess whether resin will be a suitable metal substitute for gas distribution facilities and gas equipment parts vary greatly. Table 1 compares the different conditions under which gas pipes and hot-water pipes are exposed. Resin used for hot-water pipes are subjected to harsh conditions, such as fluidity and high temperatures. Deciding whether a certain type of resin is suitable requires knowledge of all the properties of that type of resin, proper criteria to determine whether those parts are being used in an environment best suited for them, and appropriate testing methods.

Fig. 11 details that process. We design and create metal parts and their resin counterparts as the test production, and are conducting destructive tests to see if resin has enough potential as a metal substitute. Failed prototypes undergo stress analysis by finite element method, and are redesigned and correct the metal die until they pass. Then, after a mold is complete, the product is tested. This process gets repeated over and over, until finally, a product passes the criteria.

Throughout this loop, resin manufacturers, mold makers, product testers and analysts necessarily work closely together, exchanging information to create a superior final product. Fig. 12 shows how the four groups work together. 
Table 1 Comparing conditions: gas pipes vs hot-water pipes.

\begin{tabular}{llll}
\hline & Fluid & Temperature & Environment \\
\hline Gas pipe & Gas & Low & Mild \\
Hot-water pipe & Hot water & High & Hard \\
\hline
\end{tabular}

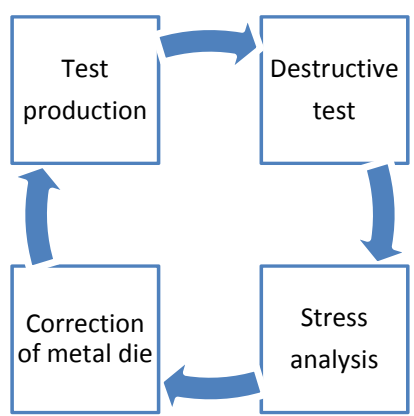

Fig. 11 The Product development loop.

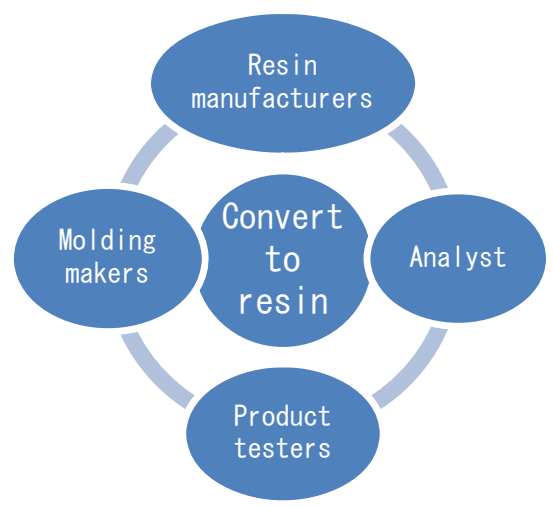

Fig. 12 Working as a team to create resin.

\section{Results and Discussion}

The most important factors in product testing and assessment criteria are that they are appropriate for the specific product and the environment in which it will be used. For example, parts that will be used in hot water must undergo a water-hammer test or stress rupture test in high temperature and high pressure, and under running water to determine its viability as a metal substitute.

Furthermore, in recent years, parts that have already undergone a switch to resin are now being considered for yet another switch to cheaper resin. Fig. 13 shows PPS headers fashioned out of a different type of resin. PPS, which is shown in Fig. 9, is super engineering plastic. It is a high-performance resin, but also costly. The number of star icons indicates the cost of the resin in Fig. 13. We are in the process of determining whether general engineering plastic or general-purpose resin are suitable replacements for PPS. The melting point for PPS is $280^{\circ} \mathrm{C}$, but for general engineering plastic, the melting point is at most $150^{\circ} \mathrm{C}$. The highest specification for Japanese hot-water dispensers is $90{ }^{\circ} \mathrm{C}$ with a boiler pressure of $0.3 \mathrm{MPa}$ while the water is running. The ability to withstand chlorination levels of $1 \mathrm{ppm}$ is required for sanitation purposes. General engineering plastic can easily satisfy these criteria, but the standards applied to testing PPS against metal cannot be applied to test general engineering plastics a viable substitute for PPS. That is because under those testing conditions, engineering plastic would melt. New assessment criteria are required.

Table 2 shows a list of testing criteria that were considered. Typically, a short-running test and a long-running test that measure endurance take place at the same time for each category. For example, when testing for stress rupture, the short-run test is conducted under low temperatures and low pressure. It is common for longer-running tests to be conducted under higher temperatures and high pressure. However, general engineering plastic has a relatively low melting point, which means it cannot be tested under the same high-temperature conditions as super engineering plastic. Therefore, when testing for stress rupture, the temperatures are lowered and conducted at lower pressure for the long-running test. Short-running tests, which usually are done under low temperature, low

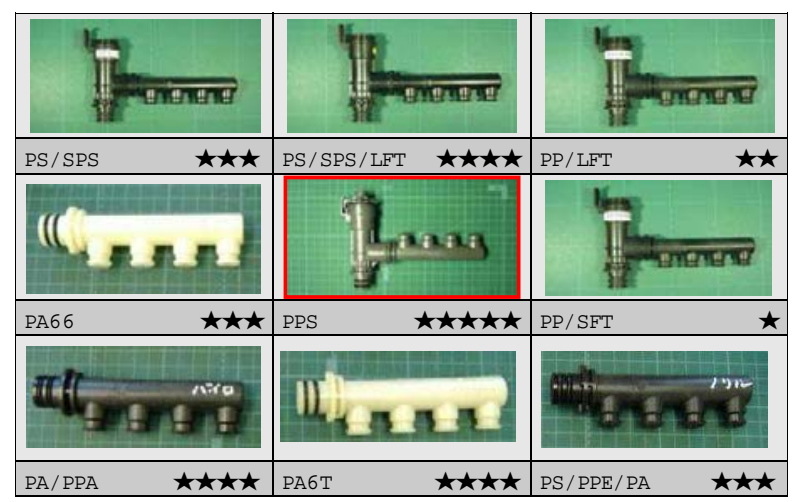

Fig. 13 Examination of PPS substitute resin. 
Table 2 Regular engineering plastic assessment criteria.

\begin{tabular}{|c|c|c|c|c|}
\hline \multirow{2}{*}{ Test } & & Metal & Super engineering plastic (PPS) & Regular engineering plastic \\
\hline & & Heat resistance & $>200^{\circ} \mathrm{C}$ & $<150^{\circ} \mathrm{C}$ \\
\hline \multirow{2}{*}{ Stress rupture } & Short-running & $150^{\circ} \mathrm{C}, 0.5 \mathrm{MPa} \rightarrow 1,000 \mathrm{~h}$ & & $130^{\circ} \mathrm{C}, 0.3 \mathrm{MPa} \rightarrow 1,000 \mathrm{~h}$ \\
\hline & Long-running & $130^{\circ} \mathrm{C}, 0.3 \mathrm{MPa} \rightarrow 8,760 \mathrm{~h}$ & & $110^{\circ} \mathrm{C}, 0.5 \mathrm{MPa} \rightarrow 8,760 \mathrm{~h}$ \\
\hline Water-hammer & & $60^{\circ} \mathrm{C}, 2 \mathrm{MPa} \rightarrow 8,760 \mathrm{~h}$ & & $80^{\circ} \mathrm{C}, 2 \mathrm{MPa} \rightarrow 8,760 \mathrm{~h}$ \\
\hline
\end{tabular}

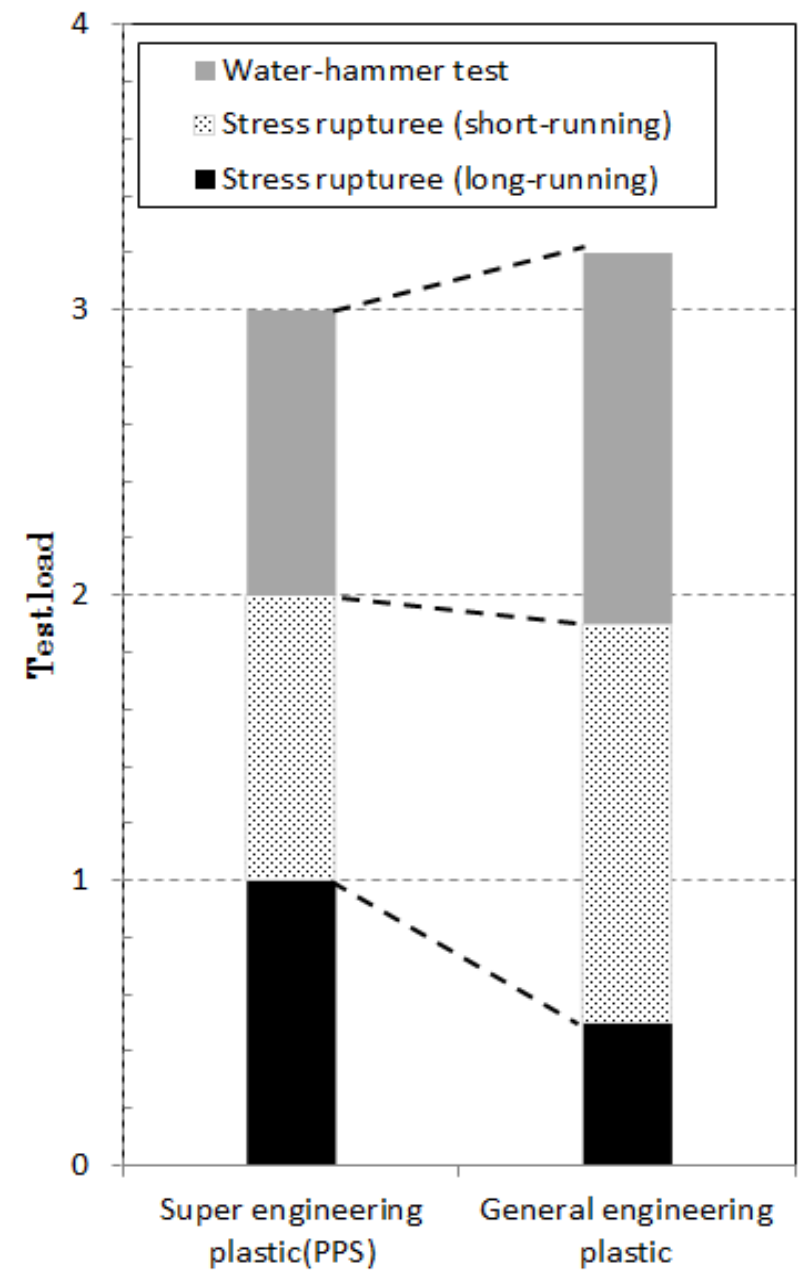

Fig. 14 Comparing test loads.

pressure conditions, are conducted under low temperatures, but higher pressure. This ended up raising the short-running testing standards for measuring pressure above the long-running testing standards. Raising the temperature during the water-hammer test for general engineering plastic above testing conditions required for super engineering plastic, we set the conditions to assess both water-hammer and long-term heat resistance.

Next, we studied the validity of these standards. Fig. 14 compares the load ratios of super engineering plastic and general engineering plastic in stress rupture and water-hammer test. Load ratio refers to the accumulation of temperature, pressure and time. The total test load for general engineering plastic was set up to surpass that of super engineering plastic. For resin with a low-melting point that complicates high-temperature endurance testing, altering the standards for pressure and time make short-term endurance testing possible by simply maintaining test loads.

\section{Conclusions}

The switch from metal to resin solves corrosion problems and reduces costs in gas piping systems. However, it is difficult to determine whether to use resin for hot water piping systems because they are exposed to harsher conditions compared to gas piping systems. We decided the criteria for using resin for hot water piping systems by using three kinds of test conditions: water-hammer, short-running stress rupture, and long-running stress rupture tests. Within this method, we compared the total sum by multiplying the test temperatures and testing times of the three tests for the resins considered as a substitute to that of super engineering plastics. By setting the test conditions for the total sum of the resins considered as a substitute either equal to or greater than those of super engineering plastics, the success of the resin can be determined through these three tests.

\section{References}

[1] Puglia, D., Biagiotti, J. and Kenny, J. M. 2004. “A Review on Natural Fiber-Based Composites-Part II: Application of Natural Reinforcements in Composite Materials for Automotive Industry." Journal of Natural 
Fibers 1 (3): 23-65.

[2] Ackley, R. 1976. "XMC \& HMC Structural Molding Compound." SAETechnical Paper 760053.

[3] Ayrilmis, N., Jarusombuti, S., Fueangvivat, V., Nauchongkol, P. and White, R. H. 2011. "Coir Fiber Reinforced Polypropylene Composite Panel for Automotive Interior Applications" Fibers and Polymers 12 (7): 919-26.

[4] Lancaster, J. K. 1967. "Composite Self-lubricating Bearing Materials.” Proc. Inst. Mech. Eng. 182 (1): 33-54.
[5] Hoagland, A. S. 1976. "Magnetic Recording Storage." IEEE Trans. Computers C-25 (12): 1283-8.

[6] Nohsho, H., Seino, I., Kajio, Y. and Sasakura, Y. 1981. "Application Technology of Plastics to Automotive Components." SAE Technical Paper 811382.

[7] Troughton, M. 2014. Standards Activities and Market Trends in Polyethylene Gas Pipe. TWI Report 23808/1/14.

[8] The Japan Gas Association. 2014. "The Spread of Polyethylene Pipes in Japan." Environmentally Friendly city Gas 6. 